\title{
Approximate Controllability of Fractional Order Retarded Semilinear Control Systems
}

\author{
Simegne Tafesse ${ }^{1}$, Nagarajan Sukavanam ${ }^{2}$ \\ ${ }^{1}$ Department of Mathematics, Haramaya University, Dire Dawa, Ethiopia \\ ${ }^{2}$ Department of Mathematics, Indian Institute Technology Roorkee (IITR), Roorkee, India \\ Email: wtsimegne@gmail.com
}

Received June 4, 2013; revised July 6, 2013; accepted July 25, 2013

Copyright (c) 2013 Simegne Tafesse, Nagarajan Sukavanam. This is an open access article distributed under the Creative Commons Attribution License, which permits unrestricted use, distribution, and reproduction in any medium, provided the original work is properly cited.

\begin{abstract}
In this paper, approximate controllability of fractional order retarded semilinear systems is studied when the nonlinear term satisfies the newly formulated bounded integral contractor-type conditions. We have shown the existence and uniqueness of the mild solution for the fractional order retarded semilinear systems using an iterative procedure approach. Finally, we obtain the approximate controllability results of the system under simple condition.
\end{abstract}

Keywords: Approximate Controllability; Fractional Order; Existence and Uniqueness, Retarded; Semilinear System; Integral Contractor

\section{Introduction}

Let $X$ and $U$ be Hilbert spaces with the corresponding function spaces $Z=L_{2}(0, \tau: X)$ and $Y=L_{2}(0, \tau: U)$

respectively. Consider the following fractional order semilinear system

$$
\begin{aligned}
& { }^{C} D_{t}^{q} x(t)=A x(t)+B u(t)+f\left(t, x_{t}\right), 0 \leq t \leq \tau \\
& x(\theta)=\phi(\theta), \quad \theta \in[-h, 0]
\end{aligned}
$$

where ${ }^{C} D_{t}^{q} x$ is the fractional order $q^{\text {th }}$ derivative in Caputo's sense, $\frac{1}{2}<q<1, A$ is the infinitesimal generator of a Co-semigroup $T(t)$ of bounded linear operator on the Hilbert space $X, B$ is a bounded linear operator from $Y \rightarrow Z \quad, \quad f$ is a nonlinear function such that $f:[0, \tau] \times C[-h, 0: X] \rightarrow X, \quad x_{t}:[-h, 0] \rightarrow X \quad$ is defined as $x_{t}(\theta)=x(t+\theta)$ for $\theta \in[-h, 0]$ and $\phi \in C(-h, 0: X)$. The norm in $X$ shall be denoted by $\|$.$\| .$

The corresponding linear fractional order system is given by

$$
\begin{aligned}
& { }^{C} D_{t}^{q} x(t)=A x(t)+B u(t), 0 \leq t \leq \tau \\
& x(\theta)=\phi(\theta), \quad \theta \in[-h, 0]
\end{aligned}
$$

Fractional differential equations are the generalization of ordinary differential equations of arbitrary non integer orders. The fractional calculus is widely popular in the field of engineering and sciences, Shantanu [1]. Debnath [2] studied the recent applications of fractional calculus to dynamical systems in control theory, electrical circuits with fractance, generalized voltage divider, viscoelasticity etc. Many papers have appeared on the controllability concepts for fractional order differential systems. For instance, Wang and Zhou [3] studied complete controllability of fractional evolution systems. In that paper fractional calculus method and fixed point theorem are used. The semigroup operator is assumed to be noncompact. Similarly controllability of fractional order impulsive neutral functional infinite delay integrodifferential systems in Banach space is studied by Tai and Wang [4]. Sakthivel et al. [5] discussed the controllability of a class of control systems governed by the semilinear fractional equations in Hilbert spaces using fixed point techniques. Kumar and Sukavanam [6] studied approximate controllability of fractional order semilinear systems with bounded delay. In that paper contraction principle and Schauder fixed point theorem are used. Zhou and Jiao [7] and El-Borai [8] studied the existence of mild solutions for fractional neutral evolution systems.

The notion of integral contractor was first introduced by Altman [9] and later on it was used by many authors to study the existence and uniqueness of solution of nonlinear evolution systems. In [10] George et al. studied the existence and uniqueness of the solution and the controllability of the nonlinear third order dispersion equa- 
tion without delay using the bounded integral contractor.

In this paper the approximate controllability of a fractional order retarded semilinear system is studied. We consider the system with the nonlinear term satisfying a bounded regular integral contractor-type condition. Under this condition we show first the existence and uniqueness of the mild solution of the system. Then using some simple condition we obtain the approximate controllability results.

\section{Preliminaries and Basic Assumptions}

Some notions of fractional order differential equations are given as follows.

Definition 2.1:

1) The fractional integral of order $\alpha$ for a function $f$ is defined as

$$
{ }_{0} I_{t}^{\alpha} f(t)=\frac{1}{\Gamma(\alpha)} \int_{0}^{t}(t-s)^{\alpha-1} f(s) \mathrm{d} s, \quad t>0, \alpha>0
$$

provided that the right hand side is defined pointwise on $[0, \infty)$. Here $\Gamma$ is the gamma function. ${ }_{0} I_{t}^{\alpha} f(t)$ is called Reimann Liouvilli integration.

2) Riemann-Liouville derivative order of $\alpha$ for a function $f:[0, \infty) \rightarrow R$ can be written as

$$
{ }_{0} D_{t}^{\alpha} f(t)=\frac{1}{\Gamma(m-\alpha)} \frac{\mathrm{d}^{m}}{\mathrm{~d} t^{m}} \int_{0}^{t}(t-s)^{m-\alpha-1} f(s) \mathrm{d} s, t>0, m-1 \leq \alpha<m
$$

3) Let $f \in C^{m}[0, \infty)$. Then the Caputo derivative of order $\alpha$ for a function $f:[0, \infty) \rightarrow R$ can be written as

$$
{ }^{c} D_{t}^{\alpha} f(t)=\frac{1}{\Gamma(m-\alpha)} \int_{0}^{t}(t-s)^{m-\alpha-1} f^{m}(s) \mathrm{d} s=I^{m-\alpha} f^{m}(t), \quad t>0,0 \leq m-1 \leq \alpha<m
$$

Define the mild solution of (1.1) as [7]

$$
x(t)=\left\{\begin{array}{lc}
S_{q}(t) \phi(0)+\int_{0}^{t}(t-s)^{q-1} T_{q}(t-s)(B u(s)+f(s, x(s-h))) \mathrm{d} s, & t>0, \frac{1}{2} \leq q<1 \\
\phi(t), & t \in[-h, 0]
\end{array}\right.
$$

where

$$
\begin{gathered}
S_{q}(t)=\int_{0}^{\infty} \xi_{q}(\theta) T\left(t^{q} \theta\right) \mathrm{d} \theta, T_{q}(t)=q \int_{0}^{\infty} \theta \xi_{q}(\theta) T\left(t^{q} \theta\right) \mathrm{d} \theta, \xi_{q}(\theta)=\frac{1}{q} \theta^{-1-\frac{1}{q}} \bar{w}_{q}\left(\theta^{\frac{-1}{q}}\right) \geq 0, \\
\bar{w}_{q}(\theta)=\frac{1}{\pi} \sum_{n=1}^{\infty}(-1)^{n-1} \theta^{-q n-1} \frac{\Gamma(n q+1)}{n !} \sin (n \pi q), \theta \in(0, \infty)
\end{gathered}
$$

$\xi_{q}$ is a probability density function defined on $(0, \infty)$, that is $\xi_{q}(\theta) \geq 0, \theta \in(0, \infty)$ and $\int_{0} \xi_{q}(\theta) \mathrm{d} \theta=1$,

$$
\int_{0}^{\infty} \theta \xi_{q}(\theta) \mathrm{d} \theta=\int_{0}^{\infty} \frac{1}{\theta^{q}} \bar{w}_{q}(\theta) \mathrm{d} \theta=\frac{1}{\Gamma(1+q)} \text { (see [7]) }
$$

Let $M$ be a constant such that $\|T(t)\| \leq M$ for all $t \in[0, \tau]$. Then the following Lemma stated as follows.

Lemma 2.1: [7] $S_{q}(t)$ and $T_{q}(t)$ are bounded linear operators and

$$
\left\|S_{q}(t)\right\| \leq M \text { and }\left\|T_{q}(t)\right\| \leq \frac{M q}{\Gamma(1+q)} \text { for all } t \in[0, \tau] .
$$

Definition 2.2: The system (1.1) is said to be approximately controllable over a time interval $[0, \tau]$, if for any given $x_{1} \in X$ and a constant $\varepsilon>0$, there exists a control $u$ such that the corresponding mild solution $x(t)$ of (1.1) satisfies $\left\|x(\tau)-x_{1}\right\| \leq \varepsilon$.

Let $C=C([0, \tau]: X)$ denote the Banach space of continuous functions on $J=[0, \tau]$ with the standard norm $\|x\|_{C}=\max \{\|x(t)\|: 0 \leq t \leq \tau\}$ for $x \in C$. By considering the nonlinear initial value problem of the form $\dot{x}(t)=F(t, x), 0 \leq t \leq \imath, x(0)=\zeta \quad$ in Banach space $X$ [9] introduced a bounded integral contractor in the following definition.

Definition 2.3: [9] A function $f$ is said to have a bounded integral contractor $\Gamma$ if

$\Gamma: J \times X \rightarrow B L(X)$ is a bounded operator and there exists a positive number $\gamma$ such that for any $w, y \in C$

$$
\max _{0 \leq t \leq \tau}\left\|f\left(t, w(t)+y(t)+\int_{0}^{t} \Gamma(s, w(s)) y(s) \mathrm{d} s\right)-f(t, w(t))-\Gamma(t, w(t)) y(t)\right\| \leq \gamma\|y\|_{C}
$$


Definition 2.4: [9] A bounded integral contractor $\Gamma$ is said to be regular if the integral equation

$$
z(t)=y(t)+\int_{0}^{t} \Gamma(s, w(s)) y(s) \mathrm{d} s
$$

has a solution $y$ in $C$ for every $w, z \in C$.
We define a bounded integral contractor operator $\Gamma^{q}$ for the fractional order system without delay in a similar fashion as:

Definition 2.5: Suppose $\Gamma^{q}: J \times X \rightarrow B L(X)$ is a bounded operator and there exists a positive number $\gamma$ such that for any $w, y \in Z$ we have

$$
\left\|f\left(t, w(t)+y(t)+\int_{0}^{t}(t-s)^{q-1} T_{q}(t-s) \Gamma^{q}(s, w(s)) y(s) \mathrm{d} s\right)-f(t, w(t))-\Gamma^{q}(t, w(t)) y(t)\right\| \leq \gamma\|y(t)\|(2.4)
$$

Then we say that $f$ has a bounded integral contractor $\Gamma^{q}$ with respect to the operator $T_{q}(t)$.

Definition 2.6: A bounded integral contractor $\Gamma^{q}$ is said to be regular if the integral equation

$$
z(t)=y(t)+\int_{0}^{t}(t-s)^{q-1} T_{q}(t-s) \Gamma^{q}(s, w(s)) y(s) \mathrm{d} s
$$

has a solution $y$ in $Z$ for every $w, z \in Z$. Let us assume

$$
\left\|\Gamma^{q}(t, w(t))\right\|_{L B(X)} \leq \beta_{1} \quad \forall t \in J, w \in Z
$$

Let $Z_{h}=L_{2}(-h, \tau: X)$. Now we define a new bounded integral contractor-type operator $\Gamma_{h}^{q}$ so as to make compatible with retarded system as follows.

Definition 2.7: Let $\Gamma_{h}^{q}: J \times C_{h} \rightarrow B L\left(C_{h}, X\right)$ be such that for $\theta \in[-h, 0]$

$$
\overline{\Gamma_{t}^{q}}\left(w_{t}\right) y_{t}(\theta)= \begin{cases}\int_{0}^{t+\theta}(t+\theta-s)^{q-1} T_{q}(t+\theta-s) \Gamma_{h}^{q}\left(s, w_{s}\right) y_{s} \mathrm{~d} s, & 0<t<\tau \\ 0, & t \in[-h, 0]\end{cases}
$$

If for any $w, y \in Z_{h}$

$$
\left\|f\left(t, w(t-h)+y(t-h)+\overline{\Gamma_{t}^{q}}(w) y(t)\right)-f(t, w(t-h))-\Gamma_{h}^{q}(t, w(t-h)) y(t-h)\right\| \leq \gamma\|y(t)\|,
$$

then $f$ is said to have an integral contractor-type operator $\Gamma_{h}^{q}$.

It can be seen easily if $\Gamma_{h}^{q}=0$, then $\overline{\Gamma_{h}^{q}}=0$ and $f$ is Lipschitz continuous.

Let us assume that $\left\|\Gamma_{h}^{q}\left(t, w_{t}\right)\right\|_{L B\left(C_{h}, X\right)} \leq \beta_{2}, \forall t \in J, w \in Z_{h}$ and $\beta=\max \left\{\beta_{1}, \beta_{2}\right\}$. Similar to Equation (2.5) consider the integral equation of the form

$$
z_{t}(\theta)=\left\{\begin{array}{lc}
y_{t}(\theta)+\int_{0}^{t+\theta}(t+\theta-s)^{q-1} T_{q}(t+\theta-s) \Gamma_{h}^{q}\left(s, w_{s}\right) y_{s} \mathrm{~d} s, & t+\theta \geq 0, z_{t}, w_{t} \in C_{h} \\
\phi(t+\theta), & t+\theta \in[-h, 0]
\end{array}\right.
$$

Definition 2.8: If $\Gamma_{h}^{q}$ is bounded and the integral Equation (2.8) has a solution $y_{t}$ in $C_{h}$ for every $z_{t}, w_{t} \in C_{h}$, then $\Gamma_{h}^{q}$ is called regular on $C_{h}$.

Now we assume the following conditions:

1) The semigroup $T(t), t \geq 0$, is compact and $T(t)=0$ for $t \in[-h, 0)$

2) $f$ has a bounded regular integral contractor-type $\Gamma_{h}^{q}$ on $C_{h}$ i.e.

$$
\left\|f\left(t, w_{t}\right)+y_{t}+\overline{\Gamma_{t}^{q}}\left(w_{t}\right) y_{t}-f\left(t, w_{t}\right)-\Gamma_{h}^{q}\left(t, w_{t}\right) y_{t}\right\| \leq \gamma\left\|y_{t}\right\|
$$

3) $f$ is uniformly bounded, i.e. there exists $M_{1}>0$ such that $\left\|f\left(t, x_{t}\right)\right\| \leq M_{1}$

4) The fractional order linear system corresponding to (1.1) is approximate controllable

5) For all $y \in Y$ there exists a constant $k>0$ such that $\|B y\| \geq k\|y\|$

Lemma 2.2: [7] If the assumption (1) is satisfied, then
$S_{q}(t)$ and $T_{q}(t)$ are also compact operators for every $t>0$.

\section{Main Result}

Define the solution mapping $W: Y \rightarrow Z$ by $W u=x$ where $x(t)$ is the unique mild solution of (1.1) corresponding to 
the control $u(t)$.

Lemma 3.1: The solution mapping $W$ is compact.

The procedure of the proof is quite similar to Lemma 1 in [11].

Theorem 3.1: Under the assumptions (1) and (2) the abstract fractional order semilinear system (1.1) has a unique mild solution if $\frac{M \gamma \tau^{q}}{\Gamma(1+q)}<1$.

Proof: First we show the existence of the mild solution.

Consider the following iteration procedure to produce sequences $\left\{x_{n}(t)\right\}$ and $\left\{y_{n}(t)\right\}$ in $X$. For $-h \leq t \leq \tau$

$$
\begin{gathered}
x_{0}(t)= \begin{cases}S_{q}(t) \phi(0)+\int_{0}^{t}(t-s)^{q-1} T_{q}(t-s) B u(s) \mathrm{d} s, & t>0 \\
\phi(t), & t \in[-h, 0]\end{cases} \\
y_{n}(t)= \begin{cases}x_{n}(t)-\int_{0}^{t}(t-s)^{q-1} T_{q}(t-s) f\left(s, x_{s n}(s-h)\right) \mathrm{d} s-x_{0}(t), t>0 \\
0, & t \in[-h, 0]\end{cases} \\
x_{n+1}(t)=x_{n}(t)-\int_{0}^{t}(t-s)^{q-1} T_{q}(t-s) \Gamma_{h}^{q}\left(s, x_{s n}\right) y_{s n} \mathrm{~d} s-y_{n}(t) \\
=x_{n}(t)-\int_{0}^{t}(t-s)^{q-1} T_{q}(t-s) \Gamma_{h}^{q}\left(s, x_{s n}\right) y_{s n} \mathrm{~d} s-\left(x_{n}(t)-\int_{0}^{t}(t-s)^{q-1} T_{q}(t-s) f\left(s, x_{s n}\right) \mathrm{d} s-x_{0}(t)\right) \\
=\int_{0}^{t}(t-s)^{q-1} T_{q}(t-s) f\left(s, x_{s n}\right) \mathrm{d} s-\int_{0}^{t}(t-s)^{q-1} T_{q}(t-s) \Gamma_{h}^{q}\left(s, x_{s n}\right) y_{s n} \mathrm{~d} s+x_{0}(t) \\
y_{n+1}(t)=x_{n+1}(t)-\int_{0}^{t}(t-s)^{q-1} T_{q}(t-s) f\left(s, x_{s(n+1)}\right) \mathrm{d} s-x_{0}(t) \\
=\int_{0}^{t}(t-s)^{q-1} T_{q}(t-s) f\left(s, x_{s n}\right) \mathrm{d} s-\overline{\Gamma_{t}^{q}}\left(s, x_{s n}\right) y_{s n}(t)+x_{0}(t) \\
-\int_{0}^{t}(t-s)^{q-1} T_{q}(t-s) f\left(s, x_{s(n+1)}\right) \mathrm{d} s-x_{0}(t)
\end{gathered}
$$

For every $t \in[0, \tau]$ and $x \in C$ we can define $x_{t} \in C_{h}$ such that $x_{t}(\theta)=x(t+\theta), h<\theta<0$. Hence, we consider the following formulation for the sequences $\left\{x_{t n}\right\}$ and $\left\{y_{t n}\right\}$.

$$
\begin{aligned}
x_{t 0}(\theta)= & \begin{cases}S_{q}(t) \phi(0)+\int_{0}^{t+\theta}(t+\theta-s)^{q-1} T_{q}(t+\theta-s) B u(s) \mathrm{d} s, & t+\theta>0 \\
\phi(t+\theta), & t+\theta \in[-h, 0]\end{cases} \\
& y_{t n}(\theta)=x_{t n}(\theta)-\int_{0}^{t+\theta}(t+\theta-s)^{q-1} T_{q}(t+\theta-s) f\left(s, x_{s n}\right) \mathrm{d} s-x_{t 0}(\theta) \\
& x_{t(n+1)}(\theta)=x_{t n}(\theta)-y_{t n}(\theta)-\overline{\Gamma_{t}^{q}}\left(x_{t n}\right) y_{t n}(\theta)
\end{aligned}
$$

Then from (3.3) we get

$$
\begin{aligned}
y_{n+1}(t)= & \int_{0}^{t}(t-s)^{q-1} T_{q}(t-s) f\left(s, x_{s n}\right) \mathrm{d} s-\int_{0}^{t}(t-s)^{q-1} T_{q}(t-s) \Gamma_{h}^{q}\left(s, x_{s n}\right) y_{s n} \mathrm{~d} s \\
& -\int_{0}^{t}(t-s)^{q-1} T_{q}(t-s) f\left(s, x_{s n}-y_{s n}-\overline{\Gamma_{t}^{q}}\left(x_{s n}\right) y_{s n}\right) \mathrm{d} s \\
= & -\int_{0}^{t}(t-s)^{q-1} T_{q}(t-s)\left\{f\left(s, x_{s n}-y_{s n}-\overline{\Gamma_{t}^{q}}\left(s, x_{s n}\right) y_{s n}\right)-f\left(s, x_{s n}\right)+\Gamma_{h}^{q}\left(s, x_{s n}\right) y_{s n}\right\} \mathrm{d} s
\end{aligned}
$$

Applying the definition (2.7) with $y_{t}:-y_{s n}$ and $w_{t}: x_{s n}$ we obtain the following inequality. 


$$
\begin{gathered}
\left\|y_{n+1}(t)\right\| \leq \int_{0}^{t} \mid(t-s)^{q-1}\left\|T_{q}(t-s)\right\|\left\|f\left(s, x_{s n}-y_{s n}-\overline{\Gamma_{t}^{q}}\left(x_{s n}\right) y_{s n}\right)-f\left(s, x_{s n}\right)+\Gamma_{h}^{q}\left(s, x_{s n}\right) y_{s n}\right\| \mathrm{d} s \\
=\int_{0}^{t} \mid(t-s)^{q-1}\left\|T_{q}(t-s)\right\|\left\|f\left(s, w_{t}+y_{t}+\overline{\Gamma_{t}^{q}}\left(w_{t}\right) y_{t}\right)-f\left(s, w_{t}\right)-\Gamma_{h}^{q}\left(s, w_{t}\right) y_{t}\right\| \mathrm{d} s \\
\leq \frac{M q \gamma}{\Gamma(1+q)} \int_{0}^{t}\left|(t-s)^{q-1}\right|\left\|y_{s n}\right\|_{C_{h}} \mathrm{~d} s=\frac{M q \gamma}{\Gamma(1+q)} \int_{0}^{t}\left|(t-s)^{q-1}\right| \max _{-h \leq \theta \leq 0}\left\|y_{n}(s+\theta)\right\| \mathrm{d} s \\
\max _{-h \leq t \leq \tau}\left\|y_{n+1}(t)\right\| \leq \frac{M q \gamma}{\Gamma(1+q)} \max _{h \leq t \leq \tau}\left\|y_{n}(t)\right\| \int_{0}^{t}(t-s)^{q-1} \mathrm{~d} s \\
\leq \frac{M \gamma \tau^{q}}{\Gamma(1+q)} \max _{h \leq t \leq \tau}\left\|y_{n}(t)\right\| \leq\left(\frac{M \gamma \tau^{q}}{\Gamma(1+q)}\right)^{2} \max _{-h \leq t \leq \tau}\left\|y_{n-1}(t)\right\| \\
\leq \cdots \leq\left(\frac{M \gamma \tau^{q}}{\Gamma(1+q)}\right)^{n+1} \max _{-h \leq \theta \leq 0}\left\|y_{0}(t)\right\|
\end{gathered}
$$

Since $\frac{M \gamma \tau^{q}}{\Gamma(1+q)}<1$, as $n \rightarrow \infty$ we have $\left(\frac{M \gamma \tau^{q}}{\Gamma(1+q)}\right)^{n+1} \rightarrow 0 \quad \Rightarrow \lim _{n \rightarrow \infty} y_{n+1}=0 \quad \forall t \in[-h, \tau]$

Hence the $\lim _{n \rightarrow \infty} y_{n}=0$ for all $t \in[0, \tau]$.

Now we show the convergence of the sequence $x_{n}(t)$ to the mild solution of the system (1.1). From (3.2) we have

$$
\begin{aligned}
& x_{t(n+1)}(\theta)=x_{t n}(\theta)-y_{t n}(\theta)-\left(\overline{\Gamma_{t}^{q}} x_{t n}\right) y_{t n}(\theta) \\
& x_{(n+1)}(t+\theta)=x_{n}(t+\theta)-y_{n}(t+\theta)-\left(\overline{\Gamma_{t}^{q}} x_{t n}\right) y_{t n}(\theta)
\end{aligned}
$$

Note that if $t+\theta \leq 0$ then $x_{(n+1)}(t+\theta)-x_{n}(t+\theta)=0$ and $y_{n}(t+\theta)=0$

Thus for $t+\theta>0$

$$
\begin{aligned}
& \left\|x_{n+1}(t+\theta)-x_{n}(t+\theta)\right\| \leq\left\|y_{n}(t+\theta)\right\|+\int_{0}^{t+\theta} \mid(t-s)^{q-1}\|\| T_{q}(t-s)\|\| \Gamma_{h}^{q}\left(s, x_{s n}\right)\|\| y_{s n} \|_{C_{h}} \mathrm{~d} s \\
& \leq\left\|y_{n}(t+\theta)\right\|+\int_{0}^{t+\theta} \mid(t-s)^{q-1}\left\|T_{q}(t-s)\right\|\left\|\Gamma_{h}^{q}\left(s, x_{s n}\right)\right\| \max _{-h \leq \theta \leq 0}\left\|y_{n}(s+\theta)\right\| \mathrm{d} s
\end{aligned}
$$

Define $t:=t+\theta$ then we get

$$
\begin{aligned}
& \left\|x_{n+1}(t)-x_{n}(t)\right\| \leq\left\|y_{n}(t)\right\|+\int_{0}^{t} \mid(t-s)^{q-1}\left\|T_{q}(t-s)\right\|\left\|\Gamma_{h}^{q}\left(s, x_{s n}\right)\right\| \max _{0 \leq t \leq \tau}\left\|y_{n}(t)\right\| \mathrm{d} s \\
& \leq\left\|y_{n}(t)\right\|+\frac{M q \beta}{\Gamma(1+q)} \max _{0 \leq t \leq \tau}\left\|y_{n}(t)\right\| \int_{0}^{t}\left|(t-s)^{q-1}\right| \mathrm{d} s \\
& \leq\left(1+\frac{M \beta \tau^{q}}{\Gamma(1+q)}\right)\left(\frac{M \gamma \tau^{q}}{\Gamma(1+q)}\right)^{n+1} \max _{0 \leq t \leq \tau}\left\|y_{0}(t)\right\|
\end{aligned}
$$

Consider the sequence of $\left\{x_{n}(t)\right\}$ in $X$. For a positive integers $m$ and $n$, assume $m<n$. Then from the above procedures we have

$$
\begin{aligned}
& \left\|x_{n}(t)-x_{m}(t)\right\| \\
& \leq\left\|x_{n}(t)-x_{n-1}(t)\right\|+\left\|x_{n-1}(t)-x_{n-2}(t)\right\|+\cdots+\left\|x_{m+1}(t)-x_{m}(t)\right\| \\
& \leq\left(1+\frac{M \beta \tau^{q}}{\Gamma(1+q)}\right) \max _{0 \leq t \leq \tau}\left\|y_{0}(t)\right\| \sum_{k=m}^{n-1}\left(\left(\frac{M \gamma \tau^{q}}{\Gamma(1+q)}\right)^{k+1}\right)
\end{aligned}
$$


Clearly the right hand side is the tail of a convergent series for sufficiently large $m$ and $n$ since $\frac{M \gamma \tau^{q}}{\Gamma(1+q)}<1$. Thus the sequence $x_{n}$ is a Cauchy sequence in $C$ hence the sequence converges to say $x^{\prime}$ in $X$.

Therefore from (3.1) we have

$$
\begin{aligned}
& \lim _{n \rightarrow \infty} y_{n}(t)=\lim _{n \rightarrow \infty} x_{n}(t)-\lim _{n \rightarrow \infty} \int_{0}^{t}(t-s)^{q-1} T_{q}(t-s) f\left(s, x_{s n}\right) \mathrm{d} s-x_{0}(t), t \in(0, \tau] \\
& \Rightarrow 0=x^{\prime}(t)-\int_{0}^{t}(t-s)^{q-1} T_{q}(t-s) f\left(s, x_{s}^{\prime}\right) \mathrm{d} s-x_{0}(t) \\
& \Rightarrow x^{\prime}(t)=x_{0}(t)+\int_{0}^{t}(t-s)^{q-1} T_{q}(t-s) f\left(s, x_{s}^{\prime}\right) \mathrm{d} s
\end{aligned}
$$

Hence $x^{\prime}(t)$ is a mild solution of the system (1.1).

Now let us show the uniqueness of a mild solution. Let $x_{1}(t)$ and $x_{2}(t)$ be the two mild solutions of (1.1) with control $u$. By the regularity of the integral contractor type $\Gamma_{h}^{q}$ with $z_{t}=x_{t 2}-x_{t 1}$ there exists $y_{t}$ in $C_{h}$ such that

$$
\begin{aligned}
z_{t}(\theta) & =y_{t}(\theta)+\int_{0}^{t+\theta}(t+\theta-s)^{q-1} T_{q}(t+\theta-s) \Gamma_{h}^{q}\left(s, x_{s 1}\right) y_{s} \mathrm{~d} s \\
x_{t 2}(\theta) & =x_{t 1}(\theta)+y_{t}(\theta)+\int_{0}^{t+\theta}(t+\theta-s)^{q-1} T_{q}(t+\theta-s) \Gamma_{h}^{q}\left(s, x_{s 1}\right) y_{s} \mathrm{~d} s \\
& =x_{t 1}(\theta)+y_{t}(\theta)+\overline{\Gamma_{h}^{q}}\left(x_{t 1}\right) y_{t}(\theta)
\end{aligned}
$$

But

$$
\begin{aligned}
& x_{2}(t)-x_{1}(t)=\int_{0}^{t}(t-s)^{q-1} T_{q}(t-s)\left\{f\left(s, x_{s 2}\right)-f\left(s, x_{s 1}\right)\right\} \mathrm{d} s \\
& =\int_{0}^{t}(t-s)^{q-1} T_{q}(t-s)\left\{f\left(s, x_{s 1}+y_{s}+\overline{\Gamma_{t}^{q}}\left(x_{s 1}\right) y_{s}\right)-f\left(s, x_{s 1}\right)-\Gamma_{h}^{q}\left(s, x_{s 1}\right) y_{s}\right\} \mathrm{d} s \\
& \quad+\int_{0}^{t}(t-s)^{q-1} T_{q}(t-s) \Gamma_{h}^{q}\left(s, x_{s 1}\right) y_{s} \mathrm{~d} s \\
& \left\|x_{2}(t)-x_{1}(t)\right\| \leq \int_{0}^{t}\left\|(t-s)^{q-1} T_{q}(t-s)\right\| \| f\left(s, x_{s 1}+y_{s}+\overline{\Gamma_{t}^{q}}\left(x_{s 1}\right) y_{s}\right)-f\left(s, x_{s 1}\right) \\
& \quad-\Gamma_{h}^{q}\left(s, x_{s 1}\right) y_{s}\left\|\mathrm{~d} s+\int_{0}^{t}\right\|(t-s)^{q-1} T_{q}(t-s)\|\| \Gamma_{h}^{q}\left(s, x_{s 1}\right) y_{s} \| \mathrm{d} s \\
& \leq \frac{M q \gamma}{\Gamma(1+q)} \int_{0}^{t} \mid(t-s)^{q-1}\left\|y_{s}\right\|_{C_{h}} \mathrm{~d} s+\frac{M q \beta}{\Gamma(1+q)} \int_{0}^{t}(t-s)^{q-1}\left\|y_{s}\right\|_{C_{h}} \mathrm{~d} s \\
& \leq \frac{M \tau^{q}}{\Gamma(1+q)}(\gamma+\beta) \max _{-h \leq \theta \leq 0}\left\|y_{n}(s+\theta)\right\|
\end{aligned}
$$

Note that if $\mathrm{t}+\theta \in[-\mathrm{h}, 0]$, then $x_{1}(t+\theta)=\phi(t+\theta)=x_{2}(t+\theta)$ and we put $y(t+\theta)=0$. Moreover, $x_{1}(t)=x_{t 1}(0), x_{2}(t)=x_{t 2}(0)$ and $y(t)=y_{t}(0)$.

Hence

$$
x_{2}(t)=x_{1}(t)+y(t)+\int_{0}^{t}(t-s)^{q-1} T_{q}(t-s) \Gamma_{h}^{q}\left(s, x_{s 1}\right) y_{s} \mathrm{~d} s
$$

Then we have

$$
y(t)=x_{2}(t)-x_{1}(t)-\int_{0}^{t}(t-s)^{q-1} T_{q}(t-s) \Gamma_{h}^{q}\left(s, x_{s 1}\right) y_{s} \mathrm{~d} s
$$


This implies that

$$
\begin{aligned}
\|y(t)\| & \leq \frac{M q}{\Gamma(1+q)} \int_{0}^{t}\left|(t-s)^{q-1}\right|\left\|f\left(s, x_{s 2}\right)-f\left(s, x_{s 1}\right)-\Gamma_{h}^{q}\left(s, x_{s 1}\right) y_{s}\right\| \mathrm{d} s \\
& \leq \frac{M q \gamma}{\Gamma(1+q)} \int_{0}^{t}\left|(t-s)^{q-1}\right|\left\|y_{s}\right\|_{C_{h}} \mathrm{~d} s \\
& \leq \frac{M q \gamma}{\Gamma(1+q)} \int_{0}^{t}\left|(t-s)^{q-1}\right| \max _{-h \leq \theta \leq 0}\left\|y_{n}(s+\theta)\right\| \mathrm{d} s
\end{aligned}
$$

Since the integrand in the right hand side is positive then the integral is an increasing function of $t$. Hence

$$
\max _{-h \leq r \leq t}\|y(r)\| \leq \frac{M q \gamma}{\Gamma(1+q)} \int_{0}^{t}\left|(t-s)^{q-1}\right| \max _{-h \leq s \leq t}\left\|y_{n}(s)\right\| \mathrm{d} s
$$

By Gronwall's inequality $y(t)$ becomes zero for all $t \geq$ 0 . Since

$$
\left\|y_{t}\right\|_{C_{h}}=\max _{-h \leq \theta \leq 0}\|y(t+\theta)\| \leq \max _{-h \leq t \leq \tau}\|y(t)\|
$$

This implies that $y_{t}$ is zero. Therefore, $x_{2}=x_{1}$ which means that the mild solution of (1.1) is unique. This completes the proof of the theorem.

Theorem 3.2: Consider the assumptions (1)-(5), then the abstract retarded semilinear control system (1.1) is approximate controllable.

Proof: Let $w(t)$ be the solution of the linear control system (1.2) corresponding to the control $\mathrm{v}$ and consider the following system.

$$
\begin{aligned}
& { }^{C} D_{t}^{q} x(t)=A x(t)+f\left(t, x_{t}\right)+B v(t)-f\left(t, w_{t}\right), 0 \leq t \leq \tau \\
& x(\theta)=\phi(\theta), \quad \theta \in[-h, 0]
\end{aligned}
$$

Note that the above system is the same as the system (1.1) in which $B u$ is replaced by $B v(t)-f\left(t, w_{t}\right)$. We define the mild solution of the linear system (1.2) as

$$
w(t)= \begin{cases}S_{q}(t) \phi(0)+\int_{0}^{t}(t-s)^{q-1} T_{q}(t-s) B v(s) \mathrm{d} s, & t \in[0, \tau] \\ \phi(t), & t \in[-h, 0]\end{cases}
$$

And the mild solution of the system (3.6) is

$$
x(t)= \begin{cases}S_{q}(t) \phi(0)+\int_{0}^{t}(t-s)^{q-1} T_{q}(t-s)\left(B v(s)+f\left(s, w_{s}\right)-f\left(s, x_{s}\right)\right) \mathrm{d} s, & t \in[0, \tau], \\ \phi(t), & t \in[-h, 0]\end{cases}
$$

But by the regularity condition with $z_{t}=w_{t}-x_{t}$ there exists $y_{t}$ such that

$$
\begin{aligned}
z_{t} & =w_{t}-x_{t}=y_{t}+\overline{\Gamma_{t}^{q}}\left(x_{s 1}\right) y_{s} \\
y_{t} & =w_{t}-x_{t}-\overline{\Gamma_{t}^{q}}\left(x_{s 1}\right) y_{s} \\
& =w_{t}-x_{t}-\int_{0}^{t}(t-s)^{q-1} T_{q}(t-s) \Gamma_{h}^{q}\left(s, x_{s}\right) y_{s} \mathrm{~d} s
\end{aligned}
$$

Taking the norms we get

$$
\left\|y_{t}\right\|_{C_{h}} \leq\left\|w_{t}-x_{t}\right\|_{C_{h}}-\frac{M q \beta}{\Gamma(1+q)} \int_{0}^{t}\left|(t-s)^{q-1}\right|\left\|y_{s}\right\|_{C_{h}} \mathrm{~d} s
$$

By Gronwall’s inequality

$$
\left\|y_{t}\right\|_{C_{h}} \leq\left\|w_{t}-x_{t}\right\|_{C_{h}} \exp \left(\frac{M \beta \tau^{q}}{\Gamma(1+q)}\right)
$$

Subtracting (3.8) from (3.7) and taking norm on both sides we get 


$$
\begin{aligned}
\left\|w_{t}-x_{t}\right\|_{C_{h}} \leq & \left\|\int_{0}^{t}(t-s)^{q-1} T_{q}(t-s)\left(f\left(s, w_{s}\right)-f\left(s, x_{s}\right)-\Gamma_{h}^{q}\left(s, x_{s}\right) y_{s}\right) \mathrm{d} s\right\| \\
& +\left\|\int_{0}^{t}(t-s)^{q-1} T_{q}(t-s) \Gamma_{h}^{q}\left(s, x_{s}\right) y_{s} \mathrm{~d} s\right\| \\
\leq & \frac{M q}{\Gamma(1+q)} \int_{0}^{t}(t-s)^{q-1}\left\|f\left(s, w_{s}\right)-f\left(s, x_{s}\right)-\Gamma_{h}^{q}\left(s, x_{s}\right) y_{s}\right\| \mathrm{d} s \\
& +\frac{M q \beta}{\Gamma(1+q)} \int_{0}^{t}(t-s)^{q-1}\left\|y_{s}\right\|_{C_{h}} \mathrm{~d} s \\
\leq & \frac{M q}{\Gamma(1+q)}(\gamma+\beta) \int_{0}^{t} \mid(t-s)^{q-1}\left\|y_{s}\right\|_{C_{h}} \mathrm{~d} s
\end{aligned}
$$

From (3.9) and (3.10) it follows that

$$
\begin{aligned}
\left\|w_{t}-x_{t}\right\|_{C_{h}} & \leq \frac{M q}{\Gamma(1+q)}(\gamma+\beta) \exp \left(\frac{M \beta \tau^{q}}{\Gamma(1+q)}\right) \int_{0}^{t}\left|(t-s)^{q-1}\right|\left\|w_{s}-x_{s}\right\|_{C_{h}} \mathrm{~d} s \\
& \leq \frac{M q}{\Gamma(1+q)}(\gamma+\beta) \exp \left(\frac{M \beta \tau^{q}}{\Gamma(1+q)}\right) \int_{0}^{t}\left|(t-s)^{q-1}\right| \max _{-h \leq \theta \leq 0}\|w(s+\theta)-x(s+\theta)\|_{C_{h}} \mathrm{~d} s
\end{aligned}
$$

Since the integrand in the right hand side is positive then the integral is an increasing function of $t$. Hence

$$
\sup _{0 \leq r \leq t}\left\|w_{t}-x_{t}\right\| \leq \frac{M q}{\Gamma(1+q)}(\gamma+\beta) \exp \left(\frac{M \beta \tau^{q}}{\Gamma(1+q)}\right) \int_{0}^{t}\left|(t-s)^{q-1}\right| \sup _{0 \leq s \leq t}\|w(s)-x(s)\| \mathrm{d} s
$$

Again Gronwall's inequality implies $w(t)=x(t)$ for all $t \in[-h, \tau]$. Under condition (5) the equation $B u=B v-f\left(t, x_{t}\right)$ has a solution $u(t)$. Therefore, the fractional order retarded semilinear control system (1.1) is approximately controllable with control $u$.

\section{REFERENCES}

[1] S. Das, "Functional Fractional Calculus," 2nd Edition, Springer-Verlag, Berlin, Heldelberg, 2011, doi:10.1007/978-3-642-20545-3

[2] L. Debnath, "Recent Applications of Fractional Calculus to Science and Engineering," International Journal of Mathematics and Mathematical Sciences, Vol. 2003, No. 54, 2003, pp. 3413-3442.

[3] J. R. Wang and Y. Zhou, "Complete Controllability of Fractional Evolution Systems,” Communication in Nonlinear Science and Numerical Simulation, Vol. 17, No. 11, 2012, pp. 4346-4355. doi:10.1016/j.cnsns.2012.02.029

[4] Z. Tai and X. Wang, "Controllability of Fractional Order Impulsive Neutral Functional Infinite Delay Integrodifferential Systems in Banach Spaces," Applied Mathematics Letters, Vol. 22, No. 11, 2009, pp. 1760-1765. doi:10.1016/j.aml.2009.06.017

[5] R. Sakthivel, Y. Ren and N. I. Mahmudov, "On the Approximate Controllability of Semilinear Fractional Differential Systems," Computers and Mathematics with Ap- plications, Vol. 62, No. 3, 2011, pp. 1451-1459.

[6] S. Kumar and N. Sukavanam, "Approximate Controllability of Fractional Order Semilinear Systems with Bounded Delay,” Journal of Differential Equations, Vol. 252, No. 11, 2012, pp. 6163-6174. doi:10.1016/j.jde.2012.02.014

[7] Y. Zhou and F. Jiao, "Existence of Mild Solutions for Fractional Neutral Evolutions," Computers \& Mathematics with Applications, Vol. 59, No. 3, 2010, pp. 10631077. doi:10.1016/j.camwa.2009.06.026

[8] M. M. El-Borai, "Probability Densities and Fundamental Solutions of Fractional Evolution Equations," Chaos Solitons and Fractals, Vol. 14, No. 3, 2002, pp. 433-440. doi:10.1016/S0960-0779(01)00208-9

[9] M. Altman, "Inverse Differentiability Contractors and Equations in Banach Spaces,” Studia Mathematica, Vol. 46, No. 1, 1973, pp. 1-15.

[10] R. K. George, D. N. Chalishajar and A. K. Nandakumaran, "Exact Controllability of the Nonlinear Third-Order Dispersion Equation,” Journal of Mathematical Analysis and Applications, Vol. 332, No. 2, 2007 pp. 1028-1044. doi:10.1016/j.jmaa.2006.10.084

[11] L. Wang, “Approximate Controllability of Delayed Semilinear Control Systems,” Journal of Applied Mathematics and Stochastic Analysis, Vol. 2005, No. 1, 2005, pp. 67-76. 